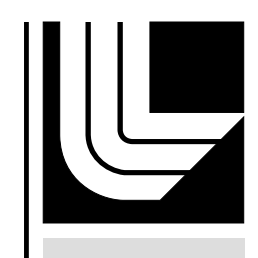

LAWRENCE LIVERM ORE N A T IO N A L LABORATORY
Relating the Proca Photon Mass and the Cosmic Vector Potential via Solar Wind

D. D. Ryutov

November 9, 2009

Physical Review Letters 
This document was prepared as an account of work sponsored by an agency of the United States government. Neither the United States government nor Lawrence Livermore National Security, LLC, nor any of their employees makes any warranty, expressed or implied, or assumes any legal liability or responsibility for the accuracy, completeness, or usefulness of any information, apparatus, product, or process disclosed, or represents that its use would not infringe privately owned rights. Reference herein to any specific commercial product, process, or service by trade name, trademark, manufacturer, or otherwise does not necessarily constitute or imply its endorsement, recommendation, or favoring by the United States government or Lawrence Livermore National Security, LLC. The views and opinions of authors expressed herein do not necessarily state or reflect those of the United States government or Lawrence Livermore National Security, LLC, and shall not be used for advertising or product endorsement purposes. 


\title{
Relating the Proca photon mass and the cosmic vector potential via solar wind
}

D.D. Ryutov

Lawrence Livermore National Laboratory, Livermore, CA 94551, USA

\begin{abstract}
The effect of the Proca photon mass $m_{p h}$ and cosmic vector potential $A_{C}$ on the dynamics of the Solar wind is considered. For large-enough values of the parameter $A_{C} m_{p h}^{2}$, the Solar wind structure at the distance $\sim 40$ AU from the Sun would change significantly with respect to the actually observed flow. Absence of such deviations gives an upper bound on the parameter $A_{C} m_{p h}^{2} 9$ orders of magnitude less than in laboratory experiments measuring torque on a toroidal magnet.
\end{abstract}

PACS Numbers: 03.50De, 14.70.Bb, 96.50.Bh

A nonzero fixed photon mass would make the vector potential of the magnetic field an observable quantity having a direct dynamical impact on the conducting media. In this paper we consider the possible effect of the vector potential produced by the currents at the Galactic and extra-Galactic scales ("cosmic" vector potential) on the Solar wind at large distances from the Sun. Thus we study dynamics of the largest "test body" available in the Solar system, the Solar wind plasma filling the whole heliosphere.

The currently accepted upper bound on the photon mass [1] is impressively small, $m_{p h}<1.5 \times 10^{-51} \mathrm{~g}$, or $1.5 \times 10^{-24}$ of the electron mass. Still, even at this level, the finiteness of the photon mass would have a significant effect on electromagnetic phenomena occurring at the scales exceeding the photon Compton length $\lambda=\hbar / m_{p h} c$, with $m_{p h}, c$ and $\hbar$ being the photon mass, the speed of light, and the Planck constant, respectively. For the currently accepted value of $m_{p h}$, one has $\lambda \approx 1.5 \times 10^{13} \mathrm{~cm}$.

The interrelation between the finite (albeit small) photon mass and large-scale natural electromagnetic phenomena has been recognized decades ago by E. Schroedinger [2] who 
suggested to use measurements of the geomagnetic field to look for possible deviations from the standard electrodynamics and concluded that $\lambda>10^{4} \mathrm{~km}$ (i.e., $m_{p h}<3 \times 10^{-47} \mathrm{~g}$ ). Later, the measurements of the magnetic field of Jupiter by the Pioneer 10 spacecraft, allowed lowering the mass limit to $10^{-48} \mathrm{~g}$, this corresponding to $\lambda \sim 3 \times 10^{10} \mathrm{~cm}[3]$.

Going to even greater scales means that one will have to account for the presence of the ambient plasma, which, generally speaking, carries non-negligible currents. Therefore, getting to larger scales makes it necessary to analyze a coupled system of a magnetic field and a plasma, and the dynamics of the conducting medium becomes an inseparable part of the problem, see, e.g., Refs. [4-6]; see also insightful reviews by Goldhaber and Nieto [7, 8].

In the classical domain (as relevant to the subject of this paper), the finiteness of the photon mass manifests itself in the change of the Ampere law, which now becomes:

$$
\nabla \times B+\frac{A}{\hbar^{2}}=\frac{4 \pi}{c} j
$$

with $\boldsymbol{B}, \boldsymbol{A}$ and $\boldsymbol{j}$ being the magnetic field, the vector potential, and the current density, respectively, and $\boldsymbol{B}=\nabla \times \boldsymbol{A}$. The displacement current is neglected, as we are concerned with deeply non-relativistic systems. Eq. (1) is a consequence of the Proca equations, describing a massive photon [9]; see also Refs. [7, 8, 10, 11]. The finite photon mass enters the problem via the second term in the left-hand side (1.h.s.) of Eq. (1): for the zero-mass photon $(\lambda \rightarrow \infty)$ we recover the standard Ampere law. This correction can be cast in terms of a "pseudocurrent" $\boldsymbol{j}_{P} \equiv-c \boldsymbol{A} / 4 \pi \lambda^{2}$ so called because it contributes like a regular current to $\nabla \times \boldsymbol{B}$ (see Refs. $[7,8]$ ).

The currently accepted bound on the photon mass [1] was obtained in Ref. [12] by the analysis of the solar wind data at the Pluto orbit collected by the Voyager 1 and 2 missions. The approach used in Ref. [12] was as follows: Based on the knowledge of the global magnetic field of the Solar wind, one could evaluate its vector potential $\boldsymbol{A}$ and - via Eq. (1) - the current $\boldsymbol{j}$ 
required to sustain the observed magnetic field $\boldsymbol{B}$. This is particularly simple for the zone well beyond the Earth orbit, where the average $\boldsymbol{B}$ is almost entirely azimuthal. The latter was predicted in the original Parker model [13] and confirmed up to the distances of tens of astronomical units by a number of space missions [14-16]. The average flow in this zone is strongly supersonic and essentially radial, with the average velocity in the equatorial region $\mathrm{v}_{l} \approx 450 \mathrm{~km} / \mathrm{s}$ independent of the distance ("ballistic" flow).

For a large-enough photon mass (small-enough $\lambda$ ), the second term in the 1.h.s. of Eq. (1) becomes dominant, and $\boldsymbol{j}$ becomes much larger than in the case of a massless photon. This leads to an increase of the $\boldsymbol{j} \times \boldsymbol{B}$ force compared to the $m_{p h}=0$ case. For a large-enough value of $m_{p h}$ (small enough $\lambda$ ), deviations from the observed flow structure would become grossly incompatible with the really observed situation, thereby setting the upper bound on the photon mass. This then yielded an upper bound for $m_{p h}$ at the level of $m_{p h}<1.5 \times 10^{-51} \mathrm{~g}$ [12].

By applying similar arguments to larger astrophysical systems, e.g., to tenuous interstellar plasmas in galaxies, one might hope to significantly improve the estimate. This approach was taken in Refs. $[17,18]$ where the limit was given as $\sim 10^{-59} \mathrm{~g}$. There was, however, no analysis presented in these papers as to what specific changes in the observational data would occur should the mass be actually much higher, say, $10^{-57} \mathrm{~g}$. Identifying such changes is a nontrivial task, given that the interstellar gas is continuously "recycled" by much heavier and energy-rich constituents of our galaxy, the stars and dense molecular clouds with embedded protostars (for details and further references see Ref. [19]). In the same direction act effects of the fine structure of the magnetic field, completely ignored in Refs. [17, 18]. All this makes it difficult to obtain a "hard" upper bound on the photon mass based on the arguments of Refs. [17, 18]. On the other hand, improved observations may sometime change this situation [8]. 
Another approach to the assessment of possible effects of large scale magnetic field (Galactic and beyond) is based on the use of an interesting consequence of Eq. (1): as was shown by R. Lakes [20], the presence of the second term in the 1.h.s. leads to the appearance of a torque acting on a current-carrying solenoid. Measuring this torque in a laboratory experiment, one can, in principle, evaluate the magnitude of the term $A_{C} / \lambda^{2}$. The measurement technique developed by Lakes was based on a special highly sensitive rotational torsion balance. In Ref. [20], the upper bound on $A_{\mathrm{C}} / \lambda^{2}$ was established at the level of $2 \cdot 10^{-7} \mathrm{G} / \mathrm{cm}$. Further refinements in the torsion balance approach have led to the lowering of the upper bound to $10^{-9} \mathrm{G} / \mathrm{cm}[21]$.

Constraining the parameter $A_{C} m_{p h}^{2}$ (or, equivalently, $A_{C} / \lambda^{2}$ ) does not allow one to independently constrain either $A_{c}$ or $m_{p h}$. However, this parameter is interesting on its own. In particular, should it be found finite, it would signal the presence of a finite Proca mass, although the mass itself would remain undetermined. The results of our paper (see below) raise the bar on the limitation of this parameter by 9 orders of magnitude compared to the previous best estimate [21].

Note that, in the analysis of Ref. [12] briefly summarized above, it was assumed that the contribution of the distant (Galactic and beyond) currents to the vector potential in the heliosphere is negligible, and the vector potential is entirely determined by the heliospheric magnetic field. A rough estimate of the heliospheric vector potential at the distances $\sim 30-40$ A.U. from the Sun would be $A_{H} \sim 10^{9} \mathrm{G} \cdot \mathrm{cm}$. In order to have significant impact on the Solar wind, the cosmic contribution to the vector potential, $A_{C}$, should exceed this value.

In this paper, we consider the effect of a possible larger vector-potential of the cosmic fields, $A_{C}>A_{H}$, on the dynamics of the Solar wind at the distances $\sim$ tens of A.U. The contribution of the cosmic magnetic fields to the vector potential $\boldsymbol{A}$ inside the heliosphere should be almost 
perfectly uniform, because of the very large scale of the cosmic field: the expected characteristic spatial scale of the Galactic field is $L_{C} \sim 1 \mathrm{kpc}$, so that the variation of the vector potential over the heliosphere $\left(L_{H^{\sim}} \sim 10^{10} \mathrm{~km}\right)$ would be $\sim L_{H} / L_{C}<10^{-6}$ and even smaller for more distant (extragalactic) sources.

The momentum equation for the solar wind at large distances from the Sun is:

$$
\rho(\mathbf{v} \cdot \nabla \mathbf{v})=\boldsymbol{j} \times \boldsymbol{B} / c \equiv \boldsymbol{f}
$$

We neglect the pressure term, given that the flow is highly supersonic. For the reference case where the photon mass is zero, the current is determined by the standard Ampere law, $\nabla \times \boldsymbol{B}=4 \pi j / c$, and the r.h.s. of Eq. (2) can be evaluated at some distance $L$ from the Sun as $B^{2} / 8 \pi L$. On the other hand, the 1.h.s. can be evaluated as $\rho v^{2} / L$. The ratio of the 1.h.s. and the r.h.s. is, therefore, $\sim B^{2} / 8 \pi \rho \mathrm{v}^{2}$. The spacecraft data show that it is very small, less than $10^{-2}$ (e.g. Ref. [14-16] and references therein), this being consistent with the ballistic-like average flow.

In the case where the vector potential is determined by cosmic sources, the expression for the corresponding contribution to the current is just

$$
j=\mathrm{c} \boldsymbol{A}_{C} / 4 \pi \hbar^{2}
$$

and the estimate of the r.h.s. of Eq. (2) becomes $\sim A_{C} B / 4 \pi \lambda^{2}$. So long as this force remains small, the flow remains ballistic with a constant expansion velocity. This situation corresponds to a large photon Compton length (small photon mass). However, with decreasing Compton length, the additional force term may become comparable to the l.h.s. and lead to a significant change of the flow features (e.g., a systematic increase or decrease of the radial velocity between the orbits of, say, Jupiter and Pluto). At a certain level of this change, it will become incompatible with the spacecraft data, leading thereby to an upper bound for $A_{C} / \hbar^{2}$. 
The direction of the galactic vector potential is not known. We denote its components parallel and perpendicular to the rotation axis as $A_{\|}$and $A_{\perp}$, respectively. We use spherical coordinates $(r, \varphi, \theta)$ with the latitudinal angle $\theta$ measured from the equatorial plane and longitudinal angle $\varphi$ measured from the projection of the vector potential onto this plane. We denote the angle formed by the cosmic vector potential with the equatorial plane by $\theta_{C}$, so that $A_{\| /}=A_{C} \sin \vartheta_{C}, A_{\perp}=A_{C} \cos \vartheta_{C}$, where $A_{C}$ is the absolute value of the cosmic vector potential.

As the detectable velocity variation is modest, less than a factor of 2 , we look for the change of the velocity field produced by the force $f$ (Eq. (2) in a perturbative manner. In the zeroth order we ignore this force (by setting the r.h.s. in Eq. (2) to zero) and consider a purely radial flow with a constant radial velocity: $\mathrm{v}_{r}=\mathrm{v}_{0}(\vartheta) ; \mathrm{v}_{\theta}=\mathrm{v}_{\varphi}=0$, where $\mathrm{v}_{0}$ is the radial velocity at some reference radius $r_{0}$ (say, the Jupiter orbit). The density distribution is then $\rho=\rho_{0}\left(r_{0} / r\right)^{2}$.

As the magnetic field at sufficiently large radii has predominantly $\varphi$ component, the force $\boldsymbol{f}=\boldsymbol{j} \times \boldsymbol{B} / c \approx \boldsymbol{A} \times \boldsymbol{B} / 4 \pi \lambda^{2}$ has only $r$ and $\vartheta$ components,

$$
\begin{aligned}
& f_{r}=-A_{C}\left(\sin \vartheta_{C} \cos \vartheta+\cos \vartheta_{C} \sin \vartheta \cos \varphi\right) B_{\varphi} / 4 \pi \lambda^{2} \\
& f_{\vartheta}=A_{C}\left(-\sin \vartheta_{C} \sin \vartheta+\cos \vartheta_{C} \cos \vartheta \cos \varphi\right) B_{\varphi} / 4 \pi \lambda^{2}
\end{aligned}
$$

For $B_{\varphi}$ one has approximately [14]: $B_{\varphi}=B_{\varphi 0}(\vartheta)\left(r_{0} / r\right)$.

Linearizing the 1.h.s. of Eq. (2) with respect to the velocity perturbations, one finds:

$$
\begin{aligned}
& \mathrm{v}_{0 r} \frac{\partial \delta \mathrm{v}_{r}}{\partial r}+\frac{\delta \mathrm{v}_{\vartheta}}{r} \frac{\partial \mathrm{v}_{0 r}}{\partial \theta}=\frac{f_{r}}{\rho_{0}}\left(\frac{r}{r_{0}}\right)^{2} ; \\
& \mathrm{v}_{0 r} \frac{\partial\left(r \delta \mathrm{v}_{\vartheta}\right)}{r \partial r}=\frac{f_{\vartheta}}{\rho_{0}}\left(\frac{r}{r_{0}}\right)^{2} .
\end{aligned}
$$


For the perturbation of the latitudinal velocity component on the way between some radius $r_{0}$ (the radius of the Jupiter orbit) to a much larger radius $r>>r_{0}$ (the radius of the Pluto orbit) one finds from Eq. (5):

$$
\delta \mathrm{v}_{\vartheta} / \mathrm{v}_{0}=2 R\left(-\sin \vartheta_{C} \sin \vartheta+\cos \vartheta_{C} \cos \vartheta \cos \varphi\right) / 3,
$$

where

$$
R=\frac{r B_{\varphi}(\vartheta) A_{C}}{8 \pi \rho \mathrm{v}_{0}^{2} \lambda^{2}}
$$

is a dimensionless parameter that characterizes the effect of the cosmic potential on the Solar wind at a distance $r$ from the Sun $(r$ must be greater than several A.U., so that the global magnetic field would already become almost entirely azimuthal). Since $R \sim r^{2}$, the perturbation grows as $r^{2}$ with the radius.

As the most detailed Solar wind data are available for the zone of not-too-high latitudes, we will be focusing on the flow in this zone. Because of a north-south symmetry of the average flow, the term $\left|\partial \mathrm{v}_{0} / \partial \vartheta\right| / \mathrm{v}_{0}$ is small here and we neglect it in Eq. (4). This yields:

$$
\delta \mathrm{v}_{r} / \mathrm{v}_{0}=R\left(-\sin \vartheta_{C} \cos \vartheta-\cos \vartheta_{C} \sin \vartheta \cos \varphi\right),
$$

If the parameter $R$ is much less than 1 , the deviations from an unperturbed average flow with constant radial velocity are small. If, however, $R$ were large, then the whole structure of the plasma flow would have changed and become incompatible with the actual picture (see specifics below). So, the upper bound on the ratio $A_{C} / \lambda^{2}$ can be roughly determined by the condition $R<1$.

When using Eq. (8), we will be substituting parameters of the Solar wind at $r \sim 40 \mathrm{AU}$ at low to moderate latitude - an area visited by several spacecraft. The parameters in the vicinity of the equatorial plane are (e.g., [22]): $\mathrm{v}_{0}=450 \mathrm{~km} / \mathrm{s}, B_{\varphi}=2 \mu \mathrm{G}, \rho=2 \cdot 10^{-26} \mathrm{~g} / \mathrm{cm}^{3}$. For this set of parameters, the condition $R<1$ yields 


$$
A_{C} / \hbar^{2}<5 \cdot 10^{-19} \mathrm{G} / \mathrm{cm}
$$

i.e., 11 orders of magnitude lower than in Ref. [20] and 9 orders of magnitude lower than in Ref. [21]. Note that this limit is obtained on the basis of direct in situ measurements of the Solar wind and in this sense is as reliable as the limits obtained by the laboratory experiments [20, 21].

Now we provide some specifics regarding the kind of modifications of the Solar wind that would be produced by the cosmic vector potential. We start from the case where $A_{C}$ has only an axial component, i.e., $\theta_{C}=\pi / 2$. Then, according to Eqs. (6), (8), the dominant effect in the equatorial zone will be on the radial velocity. As the azimuthal magnetic field changes sign from the southern to the northern hemisphere [14-16], the radial flow will be slowed down in one hemisphere and accelerated in the other (Fig. 1a). The sign depends on the phase of the Solar cycle. As the average magnetic field of the Solar wind changes sign every 11 years, the described acceleration/deceleration effect would flip over every 11 years (Fig. 1a, dashed and dotted lines).

Consider now the case where the cosmic vector potential is parallel to the equatorial plane, so that $\theta_{C}=0$. Then, according to Eqs. (6), (8), the strongest effect in the near-equatorial region (small $\theta$, just outside the current sheet) will be experienced by the latitudinal motion. The effect varies with the longitude $\varphi$ and is maximum for $\varphi=0^{0}$ and $\varphi=180^{\circ}$, where the magnetic field of the Solar wind is perpendicular to the cosmic vector potential (for our choice of coordinates). The latitudinal velocity changes sign at the equator as shown in Fig. 1b. Dashed and dotted lines correspond to the flow that expands from the equator at $\varphi=0^{0}$ and compresses to the equator at $\varphi=180^{\circ}$, respectively. After 11 years, this pattern would flip over. $\{$ In reality, the latitudinal velocity near the equator is small [16]\}. This effect leads to a significant density increase/decrease near the equator at the corresponding longitudes. For $R>1$, this systematic change would be by more than a factor of 2 . At an arbitrary orientation of the cosmic vector 
potential, there will be a superposition of the two aforementioned effects: radial acceleration/deceleration independent of the longitude, and latitudinal compression/expansion of the flow with maxima at two diametrically-opposite longitudes.

We have argued that the condition $R \sim 1$ would already be incompatible with the observations. If, however, one wants to allow for possible ambiguities, one may leave $R$ as a free parameter and replace Eq. (9) by $A_{C} / \lambda^{2}=5 \cdot 10^{-19} R, G / \mathrm{cm}$ as an upper bound. Assuming, e.g., that even a 3-fold systematic increase of the Solar wind velocity on the way from Jupiter to Pluto remains undetected, the value of $R \sim 10$ would become compatible with the observations, leading to the corresponding 10-fold increase of the upper bound on $A_{C} / \hbar^{2}$.

It cannot be completely ruled out that analysis of the whole totality of the observational data will actually discern systematic variations of the solar wind flow compared to the canonical model based on the assumption that $m_{p h}=0$. Such an analysis would however be well outside the scope of this paper.

One might hope that direct spacecraft measurement of the current density in the Solar wind plasma might allow an independent evaluation of the parameter $A_{C} / \lambda^{2}$ via Eq. (3). This is, however, hardly possible as the current density remains too small for the values of $A_{C} / \hbar^{2}$ constrained by Eq. (9). Indeed, for $A_{C} / \lambda^{2}$ taken from Eq. (9), the current density is $1.5 \cdot 10^{-18}$ $\mathrm{A} / \mathrm{cm}^{2}$. For the particle density at the Pluto's orbit $\sim 10^{-2} \mathrm{~cm}^{-3}$, this corresponds to the relative velocity of the electrons and ions of less than $10^{3} \mathrm{~cm} / \mathrm{s}$, whereas electron thermal velocity is $10^{8} \mathrm{~cm} / \mathrm{s}$. Measuring the electron distribution function with an accuracy of $10^{-5}$ is not feasible.

The upper bound on the photon mass cannot be found without the knowledge of the cosmic vector potential $[20,23]$. The dependence of the mass limit on the cosmic vector potential is illustrated by Fig. 2. We normalize the vector potential to some reference value of 
$A_{C}^{r e f}=10^{15} \mathrm{G} \cdot \mathrm{cm}$ (which approximately corresponds to the field of $1 \mu \mathrm{G}$ at the scale of $300 \mathrm{pc}$ and can be thought of as a rough estimate of the vector potential created by the orderly magnetic field in a spiral arm of our Galaxy, if such a field is present). Using Eq. (9) and relation $\lambda=\hbar / m_{p h} c$, we then find:

$$
m_{p h}(g)<5 \cdot 10^{-55} \sqrt{R A_{C}^{\text {ref }} / A_{C}} .
$$

When $A_{C}$ becomes less than the vector potential of the Solar wind $\left(10^{-6} A_{C}^{r e f}\right)$, the mass becomes independent of $A_{C}$ and reaches the currently accepted upper bound for $m_{p h}$. We emphasize again that, without independent information about the cosmic vector potential, Eq. (10) does not allow one to improve the estimate of [12].

In summary: Based on the analysis of the effects of the cosmic vector potential $A_{C}$ on the Solar wind, an improved upper bound on the product $A_{C} m_{p h}^{2}$ has been established which is 9 orders of magnitude lower than the lowest of the previously reported values. The main results are expressed by Eqs. (9) and (10).

Prepared by LLNL under Contract DE-AC52-07NA27344. 


\section{References}

1. C. Amsler, and Particle Data Group. Phys. Letters, B667, 1, 2008.

2. E. Schroedinger. Proc. Royal Irish Academy A49, 135, 1943.

3. L. Davis, A.S. Goldhaber, M.M. Nieto. Phys. Rev. Lett. 35, 1402, 1975.

4. M.A. Gintzburg. Soviet Astronomy, AJ7, 536, 1964.

5. J.V. Hollweg. Phys. Rev. Lett., 32, 961, 1974.

6. D.D. Ryutov. Plasma Phys. Control. Fusion 39, A73, 1997.

7. A.S. Goldhaber, M.M. Nieto. Rev. Mod. Phys., 43, 277, 1971.

8. A.S. Goldhaber, M.M. Nieto. To appear in Rev. Mod. Phys, 2009.

9. A. Proca. Compt. Rend. 190 1377, 1930.

10. J.D. Jackson. Classical Electrodynamics (Chichester, Wiley), 1975.

11. L.D. Landau, E.M. Lifshitz. Quantum Theory of Fields (Oxford, Pergamon), 1987.

12. D.D. Ryutov. Plasma Phys. Contr. Fus., 49, B429, 2007.

13. E.N. Parker. Astrophys. Journ. 128, 664, 1958.

14. L.F. Burlaga, N.F. Ness, Y-M. Wang, N.R. Sheeley. Journ. Geophys. Res. 103 23723, 1998.

15. N.F. Ness, L.F. Burlaga. Journ. Geophys. Res. 106 \#A8, 15803, 2001.

16. E.J. Smith. Solar wind magnetic fields. In: “Cosmic Winds and heliosphere,” p. 425, J.R. Jokipi, C.P. Sonett, M.S. Giampapa, Eds., The University of Arizona Press, Tucson,1997.

17. G.V. Chibisov. Sov. Phys. Uspekhi 19 624, 1976.

18. E. Adelberger, G. Dvali, A. Gruzinov. Phys. Rev. Lett. $98010402,2007$.

19. D.D. Ryutov. Astrophys. Journ., 674, 976, 2008.

20. R. Lakes. Phys. Rev. Lett., 80, 1826, 1998.

21. J. Luo, L-C. Tu, Z-K. Hu, E.-J. Luan. Phys. Rev. Lett. $90081801,2003$.

22. F. Bagenal et al. Pluto's interaction with the Solar wind In: Pluto and Charon, Univ. of Arizona Press, 1997; L.F. Burlaga et al. Voyager 1 studies of HMF to 81 AU during the ascending phase of Solar cycle 23 In: Solar Wind Ten p. 39, 2002.

23. A.S. Goldhaber, M.M. Nieto. Phys. Rev. Lett. 91 149101, 2003. 

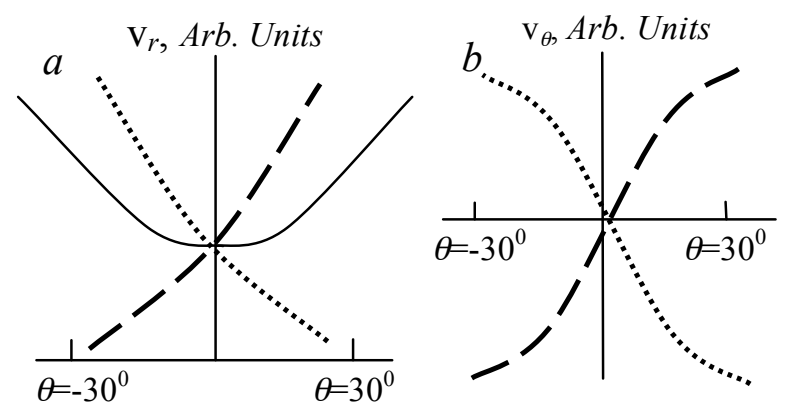

Fig. 1 A sketch of the latitudinal variation of the solar wind velocity near the heliomagnetic equator: a) Radial velocity; the solid line represents an approximately symmetric profile of the real Solar wind (e.g., [16]). The dashed line corresponds to the situation with the parameter $R$ [Eq. (7)] of order 1 and the wind accelerated in the Northern hemisphere; the dotted line corresponds to the same $R$, but to the next half-period of the Solar cycle. At higher values of $R$, the stagnation would occur in one of the hemispheres, leading to the structure completely incompatible with the observed picture. b) Latitudinal velocity. Actual latitudinal velocity is close to zero and would coincide with the horizontal axis. For $R \sim 1$, the latitudinal velocity at the distance of $30^{\circ}$ from the equator would be comparable to the radial flow velocity of $\sim 450 \mathrm{~km} / \mathrm{s}$.

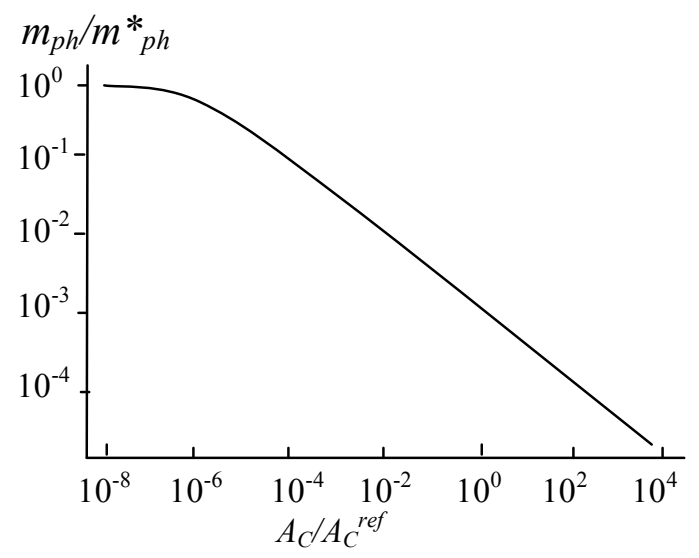

Fig. 2 The dependence of the upper bound on the photon mass (normalized to $m^{*}{ }_{p h}=1.5 \times 10^{-51} \mathrm{~g}$ ) vs. the cosmic vector potential (normalized to the reference value $A_{C}{ }^{r e f}=10^{15} \mathrm{G} \cdot \mathrm{cm}$ ), for $R=10$. At low values of $A_{C}$ the mass is determined by the solar wind vector potential and becomes independent of $A_{C}$. The allowed range of parameters $m_{p h}$ and $A_{C}$ lies below the curve. 